\title{
A Novel Approach to the Manipulation of Body-Parts Ownership Using a Bilateral Master-Slave System
}

\author{
M. Hara, Member, IEEE, G. Rognini, Student Member, IEEE, N. Evans, O. Blanke, \\ A. Yamamoto, Member, IEEE, H. Bleuler, Member, IEEE, and T. Higuchi Member, IEEE
}

\begin{abstract}
This paper introduces a novel approach to the manipulation of body-parts ownership, using the tactile rubber hand illusion (RHI) paradigm. In the conventional studies on the RHI, participant's invisible hand and the visible rubber hand are manually tapped or stroked by an experimenter. Differently, in our approach, a bilateral master-slave system is applied to provide tactile stimulations in a novel interactive manner-active self-touch. Here, we present a 3-DOF masterslave system based on human self-touch characteristics and a validation experiment using an arranged version of the conventional RHI paradigm. In this new version, participants can contact with the rubber hand by manipulating the master device with their right hand and actively touch their own left hand through the slave device. The results demonstrate that the master-slave system can be successfully used to manipulate body-parts ownership, opening up the way to new studies concerning self-touch and body representation.
\end{abstract}

\section{INTRODUCTION}

How does the human brain represent our own body and the external world surrounding us?

This question is a kind of eternal topic for human beings. Over the last decades, several efforts have been accomplished in order to understand the underlying mechanisms of body representation. Findings from neurophysiology, cognitive science, and neuroimaging have improved our knowledge about the perceptual, multisensory and sensorimotor mechanisms making our body together an extended object in the external space and the source of our sense of self (self-consciousness) [1]-[3]. Owing to these efforts, the introduction of conflicts among sensory modalities has been proven to be an effective way to experimentally manipulate the sense of ownership (self-localization) of one's own body parts.

Among these studies, rubber hand illusion (RHI), which was discovered by Botvinick and Cohen [4], has attracted the interest of many researchers. In a typical RHI experiment, a synchronous brushing of a visible rubber hand and the participants' own hand, which is hidden from their view, leads the participants to feel as if the fake hand is a part of their own body [4] [5]; an asynchronous brushing does

This work was supported by UT-EPFL joint lab project.

M. Hara, A. Yamamoto, and T. Higuchi are with Department of Precision Engineering, The University of Tokyo, 7-3-1, Japan masayuki, akio, higuchi @aml.t.u-tokyo.ac.jp

G. Rognini and $\mathrm{H}$. Bleuler are with Institute of Microengineering, Ecole Polytechnique Federale de Lausanne (EPFL), CH-1015, Switzerland giulio.rogninidepfl.ch, hannes.bleulerdepfl.ch

N. Evans and O. Blanke are with Brain Mind Institute, Ecole Polytechnique Federale de Lausanne (EPFL), CH-1015, Switzerland nathan.evansdepfl.ch, olaf.blankedepfl.ch not induce the RHI. This illusion phenomenon presents a drift of the perceived hand position toward the rubber hand, and reflects a cross-modal interaction among human vision, tactile sensation, and proprioception. The recent trend of the RHI studies is to conduct the experiment under various conditions so as to reveal all the key factors contributing to the sense of ownership for one's own body parts [6]-[12]. For example, Tsakiris and Haggard demonstrated that the RHI level is decreased when the participants gaze objects different from a fake left hand, e.g., a fake right hand or a piece of wood [8]. Costatini and Haggard examined the sensitivity of RHI by changing the orientation of rubber hand and the brushing direction [10]. According to the report by Dummer et al., the RHI level was significantly increased in active movement condition compared to in passive movement condition [11]. Further, Ehrsson et al. recently detected the brain region-premotor cortex-where is activated during the RHI by using functional magnetic resonance imaging (fMRI) [9]. In general, visual information has been considered as a dominant factor to cause the RHI. However, they also reported that the RHI can be elicited in absence of visual information by synchronously touching the right hand of blindfolded participants and guiding their left hand to touch a rubber hand [9].

As mentioned above, many researchers have used the RHI paradigm trying to shed further light on the complex multisensory and sensorimotor mechanisms underlying body representation after Botvinick and Cohen found the illusion. However, despite many insights, the comprehensive conclusions on this topic haven't been reached yet and there is still much room for discussion. A more complete knowledge may be brought by overcoming the main technical limitations of the current state of the art with robotics, haptics, and virtual reality (VR) technologies [13]. In particular, the more precise control of temporal synchrony and spatial congruency of the experimentally administered multisensory conflicts, the possibility to better manipulate active and passive touching conditions, and the contribution of haptic feedback may allow clarifying many current open questions and hypothesis about body representation in human brain.

In this paper, a novel approach with a master-slave system is proposed in order to enlarge and deepen the discussion on human body-parts ownership. The use of master-slave system enables the application of precise time delay, tapping and stroking by participants themselves (active self-touch), excellent repeatability of haptic feedback, etc., which cannot be exactly realized in the conventional methods. Hence, it is 
promising that our challenge brings a breakthrough for the studies on body ownership. First of all, this paper presents characterization of self-touch (tapping and stroking), and then, the design and validation of a master-slave system consisting of a PHANToM Omni and a 3-DOF slave device. Further, we describe an RHI experiment with an interactive (active movement) version of the tactile RHI paradigm which is the same as that used by Ehrsson [9] in order to prove that the proposed system has a possibility to induce a novel RHI. Through this approach, this paper aims at cultivating a new interdisciplinary field and presenting interactive contributions between engineering and neuroscience fields.

\section{RHI in ACtive SELF-TOUCH}

In the general RHI paradigms, an experimenter manually presents tapping or stroking stimulation on the participants' hand and the rubber hand using the experimenter's index finger or an paintbrush, as shown in Fig. 1(a). Also, there is

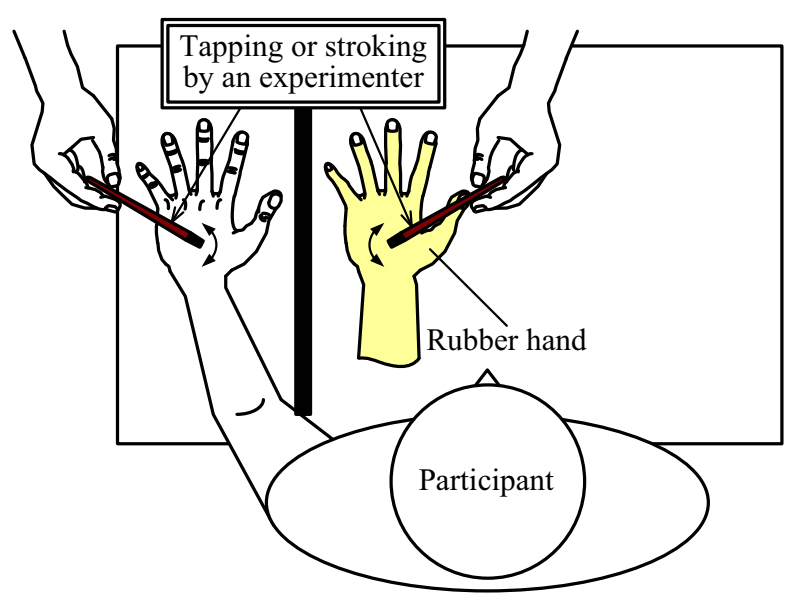

(a) Conventional condition

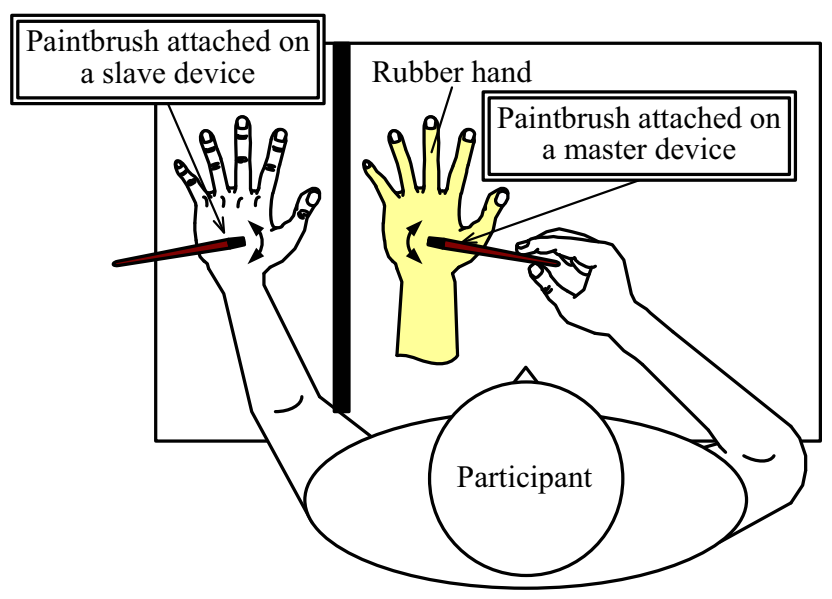

(b) Proposed condition: Active self-touch

Fig. 1. RHI conditions viewed from above. In conventional studies, participants are passively stimulated by an experimenter (a). On the other hand, the participants can interactively touch a rubber hand and their own hand using a master-slave system in the proposed condition (b). the case in which the experimenter guides the participants' hand to touch the rubber hand giving the same stimulation on their own hand [9] [12]. In these conditions, the participants' behavior is completely passive and they never move based on their will. Adding the active self-touch condition in the RHI paradigm is expected to bring a tremendous potential to further discuss the underlying mechanisms of the sense of ownership. Intuitively, we can consider, e.g., the effect of active touch that is one of the most common ways which humans use to interact with the external world and to become aware of their body in space [14] [15].

Overcoming the main limitations of the state of the art that has made active touch a hard topic to study, this paper proposes to test by using haptic technology whether active self-touch would lead to the rubber hand illusion. In our approach, a bilateral master-slave system is applied to create the situation shown in Fig. 1(b). The participants have an paintbrush attached on a master device and self-stroke a rubber hand. A slave device synchronously or asynchronously displays the same stroke with the other paintbrush on the participants' real hand. Hence, the participants can present and receive the stimulation in their own way. Our approach is not only novel (which would not have been used so far), but also includes the other noteworthy advantages. Again, since the conventional studies have used manual stimulation by an experimenter, perfectly synchronized stimulation, precise congruency of stimulated place, etc. between a real hand and the rubber hand are quite difficult to be realized. However, the application of haptic technology facilitates precise control of temporal synchrony and spatial congruency in the stimulus presentation; conversely, precise time shift, inversion of stroking direction, etc. are also possible. Once the haptic feedback and the participants' behavior are recorded during the experiment, they can be redisplayed with time advance or time delay any time. In addition, the behavioral data, which can be fetched by using sensing functions of the master-slave system, would be very useful for the analysis of RHI [16]. So, it is expected that the proposed method opens an infinite of possibilities for the study on body-parts ownership; these technical advantages would be also useful for the experiments under the conventional conditions.

\section{Characterization of Active Self-Touch}

Prior to designing the master-slave system, it is better to know the characteristics when people naturally tap or stroke their own body. In this study, tapping force and stroking speed during actively self-touching of a real hand were measured in order to grasp the characteristics.

As for the tapping force, 6 right-handed people were asked to tap anywhere they wish on the back of their left hand for $20 \mathrm{~s}$ with a stick which has a load cell (CentoNewton, EPFL-LPM) on the tip; the stick is mounted on a PHANToM Omni (SensAble), as shown in Fig. 2. In another experiment, the stroking speed was measured when the same participants stroked the back of their left hand along the middle finger for $20 \mathrm{~s}$ in their own way. The behavioral data was fetched 


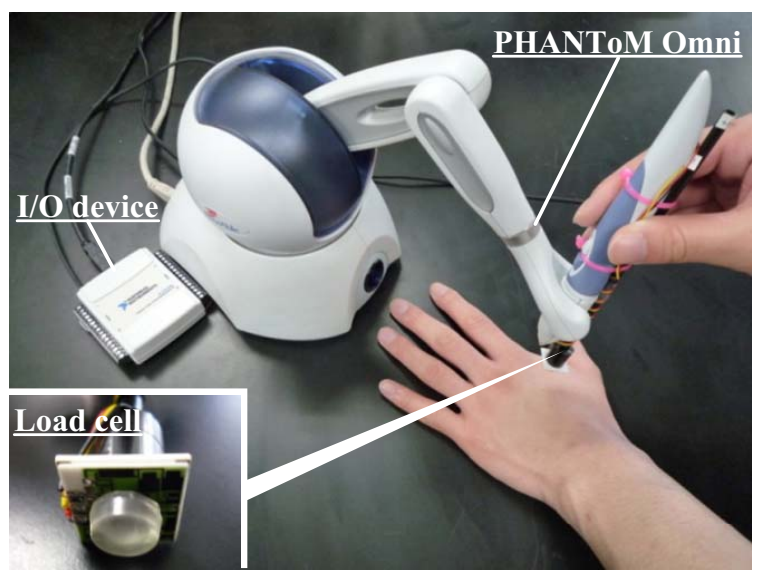

Fig. 2. An experimental apparatus for characterizing self-tapping and self-stroking. Since a load cell (CentoNewton, EPFL-LPM) linked with an I/O device (NI-6008, National Instrument) is attached at the tip of a stick mounted on PHANToM Omni (SensAble), the tapping force and the stroking manner can be observed.

TABLE I

Characterization OF Self-TaPPing

\begin{tabular}{|c|c|c|c|c|c|}
\hline & min. $F$ & max. $F$ & avg. $F$ & avg. $f$ & max. $t s$ \\
\hline \hline A & 1.97 & 6.35 & 4.16 & 2.25 & 45.7 \\
B & 1.65 & 3.25 & 2.45 & 1.05 & 24.3 \\
C & 1.97 & 5.49 & 3.73 & 1.45 & 57.4 \\
D & 2.18 & 6.99 & 4.59 & 1.30 & 26.2 \\
E & 0.58 & 2.50 & 1.54 & 1.55 & 17.2 \\
F & 2.18 & 8.38 & 5.28 & 1.90 & 38.7 \\
\hline \hline AVG & \multicolumn{5}{|c|}{3.62} \\
\hline
\end{tabular}

TABLE II

Characterization OF SELF-STROKING

\begin{tabular}{|c|c|c|c|c|}
\hline & max. $V$ & avg. $V$ & avg. $f$ & max. $s$ \\
\hline \hline A & 220.2 & 48.0 & 0.28 & 96.2 \\
B & 522.2 & 34.4 & 0.33 & 70.0 \\
C & 244.5 & 52.9 & 0.28 & 101.6 \\
D & 601.7 & 87.0 & 0.58 & 90.9 \\
E & 282.8 & 52.9 & 0.58 & 54.7 \\
F & 531.5 & 102.4 & 0.51 & 111.8 \\
\hline \hline AVG & 400.48 & 63.0 & 0.41 & 87.5 \\
\hline
\end{tabular}

$V$ : Velocity $(\mathrm{mm} / \mathrm{s}), f$ : Frequency $(\mathrm{Hz}), s:$ Stroke $(\mathrm{mm})$

using the load cell (force) and PHANToM Omni (trajectory and speed) at $1 \mathrm{~ms}$ sampling rate.

Tables I and II list the results for each experiment. Regarding the self-tapping, the average force was $3.62 \mathrm{~N}$ (Min: $0.58 \mathrm{~N}$, Max: $8.38 \mathrm{~N}$ ) and the frequency was approximately $1.58 \mathrm{~Hz}$. In this case, the stroke in the vertical direction (tapping stroke) was relatively short (approximately $34.2 \mathrm{~mm}$ ). Further, as shown in Table II, it is found that the participants stroked their hand at a speed around $63.0 \mathrm{~mm} / \mathrm{s}$ and a frequency $0.41 \mathrm{~Hz}$. These results imply that the masterslave system for active self-touch on the real hand should roughly satisfy the following performances:

- Self-tapping

- Continuous force: $4.0 \mathrm{~N}$

- Instantaneous force: $10.0 \mathrm{~N}$
- Stroke: $50.0 \mathrm{~mm}$

- Bandwidth: $1.8 \mathrm{~Hz}$

- Self-stroking

- Continuous speed: $70.0 \mathrm{~mm} / \mathrm{s}$

- Instantaneous speed: $600.0 \mathrm{~mm} / \mathrm{s}$

- Stroke: $100.0 \mathrm{~mm}$

- Bandwidth: $0.8 \mathrm{~Hz}$

These requirements are based on the behavior when people freely tapped or stroked their hand. In actual experiments, since some constraint is usually imposed on the participants' behavior to unify the experimental condition, these requirements might be more flexible.

\section{EXPERIMENTAL SYSTEM}

\section{A. Design of a 3-DOF Master-Slave System}

From past experience, the setup should be functional, but as simple as possible for such neuroscientific experiment; for example, Blakemore et al. designed a simple experimental setup with a plastic rod which enables self- and externaltickling in MRI environment [17]. In this study, a 3-DOF master-slave system was designed based on the characterization of self-touch shown in chapter III.

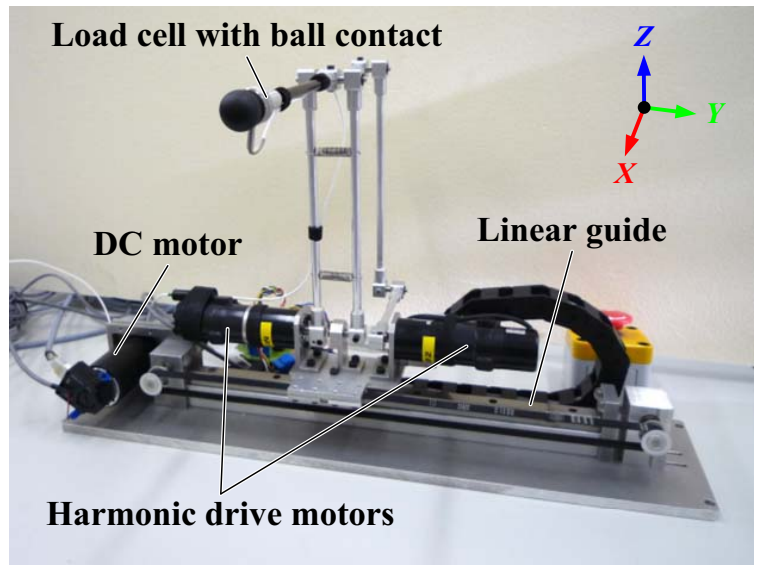

(a) 3-DOF slave device

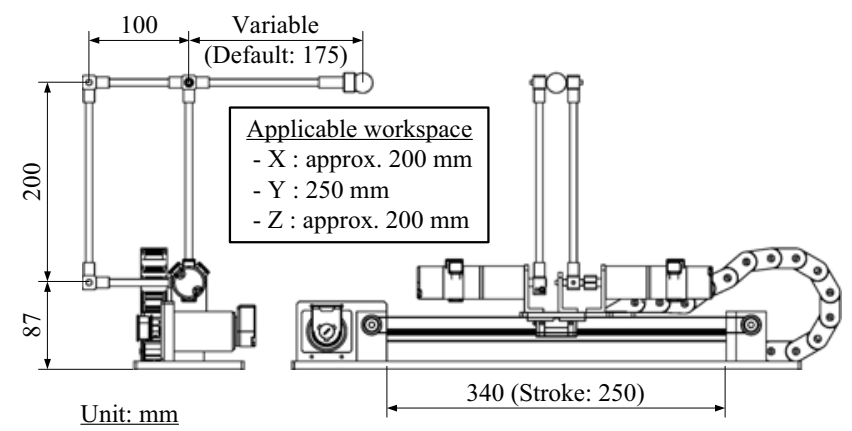

(b) Dimension

Fig. 3. A developed 3-DOF slave device. The slave device is linearly driven in $\mathrm{Y}$ direction by a toothed-belt drive mechanism using a DC motor (RE 40, Maxon). The movements in $\mathrm{X}$ and $\mathrm{Z}$ directions are generated by a parallel-link mechanism based on two harmonic drive motors (RH-8D 6006, Harmonic Drive Systems). A load cell (ELPF-TIM-50N, Measurement Specialties) is attached on the tip of the end link so as to give force feedback to the master device. 
In our system, PHANToM Omni (SensAble) was adopted as a master device for reasons of availability. Also, since the stimulation is often applied to the participants by a paintbrush in the RHI paradigm, a pen-type haptic device must be very intuitive for the participants to manipulate. Thus, we considered PHANToM Omni as the best candidate for the master device; of course, it can be replaced with other haptic device depending on the purpose.

As for the slave device, a 3-DOF tapping/stroking device was developed, as shown in Fig. 3. The device consists of two mechanisms-belt-drive mechanism and parallellink mechanism. The belt-drive mechanism is mainly for long stroking (the maximum stroke was designed to be $250 \mathrm{~mm}$ assuming the stroking of other body parts, e.g., foot), in which the belt linked with a direct-drive DC motor (RE 40, Maxon) drives a carrier on a linear guide in $\mathrm{Y}$ direction. Meanwhile, the parallel-link mechanism enables both the tapping and stroking in $\mathrm{X}$ and $\mathrm{Z}$ directions. In these directions, two harmonic drive motors (RH-8D 6006, Harmonic Drive Systems), which have a harmonic gear head of reduction ratio 50, were applied to generate sufficient tapping force. These three motors with optical encoders are connected with two kinds of motor drivers (4-Q-DC Servoamplifier LSC 30/2 \& ADS 50/5, Maxon) and the drivers receive the command voltages from a computer via PCI data acquisition cards (NI PCI-6221 \& NI PCI-6014, National Instruments). The end link-carbon-fiber tube-is removable and can be replaced with other components, e.g., a paintbrush. In the default condition, a load cell (ELPFTIM-50N, Measurement Specialties) is attached on the tip in order to feedback the contact force to the master device.

As for the software, a GUI-enabled application was programmed in Visual C++ (Microsoft). In this application, the experimenter can intuitively and quickly change the control modes and parameters of the master-slave system through a few dialog boxes and keystrokes. The experimenter can also observe all the related data (position, velocity, force, etc.) during the RHI experiment. The sampling rate was set at $1 \mathrm{~ms}$ and all the device control and data logging are performed within this sampling rate.

\section{B. Performance Evaluation}

The performance of the developed slave device was characterized so as to validate the applicability for the self-touchenabled RHI paradigm. First, the tapping forces in $\mathrm{X}$ and $\mathrm{Z}$ directions generated by the parallel-link mechanism were theoretically simulated. It depends on the device posture, but it was proven that the slave device can continuously generate at least $4.5 \mathrm{~N}$ (X direction) and $8.5 \mathrm{~N}$ (Z direction) within the workspace shown in Fig. 3(b); the instantaneous maximum forces are more than $8.5 \mathrm{~N}$ and $15.0 \mathrm{~N}$, respectively.

Further, the responsiveness of the slave device was investigated under a closed position control, in which a sinusoidal input with peak-to-peak amplitudes $100 \mathrm{~mm}$ in $\mathrm{X}$ and $\mathrm{Z}$ directions and $150 \mathrm{~mm}$ in $\mathrm{Y}$ direction were applied as the reference input. According to the frequency response analysis, the bandwidths are approximately 1.5 to $2.5 \mathrm{~Hz}$;

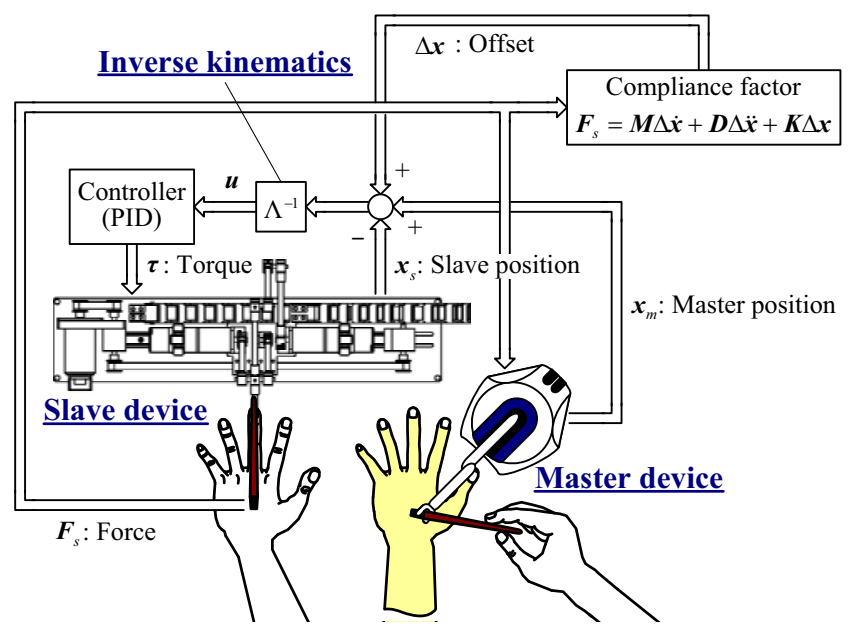

Fig. 4. A block diagram of the control system in the developed master-slave system. A force-feedback-type bilateral control system is built between the master and slave devices. On the slave side, a compliance factor can be added in order to avoid instantaneous strong contact force on one's body.

the instantaneous maximum velocity which the slave device could generate in each direction during the position control was over $900 \mathrm{~mm} / \mathrm{s}$. These results satisfy the requirements listed in chapter III, proving that the developed slave device is applicable for the self-touch-enabled RHI paradigm.

\section{Control System}

Fig. 5 illustrates a block diagram of control system implemented in the master-slave system, which is so-called force-feedback-type bilateral control system. On the master side, the force feedback when the slave device contacts with the participants' body is realized based on the contact force measured by the load cell as an input. A position control system with a PID controller is built in the slave device using 3D position of the master device as the desired position. For safety reasons, a compliance control based on the contact force is also programmed on the slave side. Thus, the input $\boldsymbol{u}$ to the PID controller is expressed as follows:

$$
\begin{gathered}
\boldsymbol{u}=\Lambda^{-1}\left(\Delta \boldsymbol{x}+\boldsymbol{x}_{m}-\boldsymbol{x}_{s}\right) \\
\Delta \boldsymbol{x}=\frac{1}{\boldsymbol{K}}\left(\boldsymbol{F}_{s}-\boldsymbol{M} \ddot{\Delta} \boldsymbol{x}-\boldsymbol{D} \dot{\Delta \boldsymbol{x}}\right)
\end{gathered}
$$

where $\boldsymbol{x}_{m}$ and $\boldsymbol{x}_{s}$ are positions of the master and slave devices, respectively. $\Delta x$ is an offset calculated from a virtual dynamics ( $\boldsymbol{M}$ : inertia, $\boldsymbol{D}$ : viscosity, $\boldsymbol{K}$ : stiffness) and the contact force $\boldsymbol{F}_{\mathrm{s}}$ on the slave side. The introduction of this compliance factor produces flexibility and prevents the slave device from applying instantaneous strong force to the participants.

In this study, the RHI paradigm with a paintbrush is used to validate the applicability of the developed master-slave system. In this case, the stroking (brushing) has only to be realized between the master and slave devices, but the tapping is not necessary. Hence, the end link of the slave device was replaced with a paintbrush and the control system without force feedback was used in the following experiment. 


\section{RHI EXPERIMENT UNDER ACtIVE SElf-Touch}

\section{A. Participants}

A total of 10 healthy right-handed participants (3 female, mean age 28 years) took part in. All participants had normal or corrected to normal vision and had no history of neurological or psychiatric conditions. All the participants gave written informed consent and were not compensated for their participation. The study protocol was approved by the local ethics research committee-La Commission d'ethique de la recherche Clinique de la Faculte de Biologie et de Medecine-at the University of Lausanne, Switzerland and was performed in accordance with the ethical standards laid down in the Declaration of Helsinki.

\section{B. Experimental Procedure}

The experimental procedure was readopted from the experiments of Ehrsson [9] and White [12]. The participants were blindfolded and seated with their arms resting on a table in a pronated position (palms down), as shown in Fig. 5. Headphones were also used to mask the environmental noise and the sound produced by their brushing. A rubber life-size prosthesis hand that imitates human left hand was placed on the table between the participant's hands $(200 \mathrm{~mm}$ to the right of the participant's left hand). The participants brushed the rubber hand by manipulating the paintbrush attached on the master device and the slave device brushed participants' left hand by reproducing the participants' movement. The stimulation was applied in two different conditionssynchronous and asynchronous. The synchronous condition was characterized by no delay between the master and slave movements. On the other hand, a delay of $500 \mathrm{~ms}$ between the master and slave movements was added during the asynchronous condition. The participants kept giving the stimulation to the rubber hand and their own hand for $60 \mathrm{~s}$ in each condition.

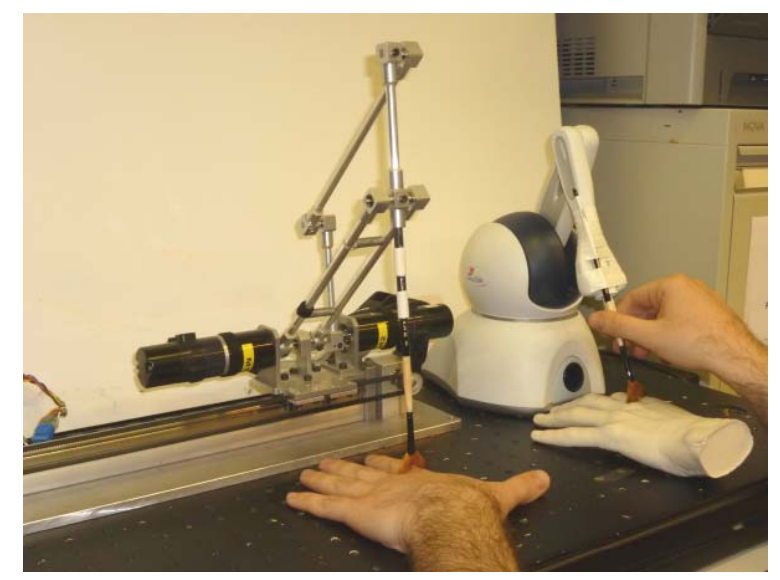

Fig. 5. Experimental environment. The master device (PHANToM Omni) and the slave device are located in front of the blindfolded participants' right and left hands, respectively. The participants manipulate a paintbrush attached on the master device to brush the rubber hand, which was located $200 \mathrm{~mm}$ right from the participant's left hand, in their own way. The slave device synchronously or asynchronously gives the same brushing on the participants' own hand.

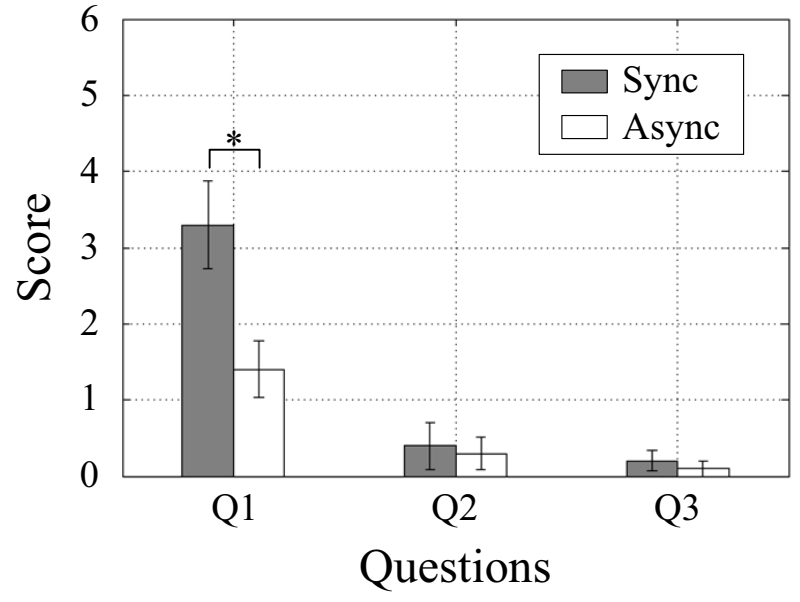

Fig. 6. Testing the RHI effect on the 10 healthy participants. Bar charts and error bars show the average scores for each statement in each condition and its standard error of the mean (SEM), respectively. The feelings of the 10 participants were assessed by means of the conventional questionnaire (Q1 to Q3) and a seven-point Likert scale. Only for Q1 score statistically indicates the significant difference $(p<0.05)$ between synchronous and asynchronous conditions.

Just after the self-brushing under each condition, the participants completed a "rubber-hand illusion questionnaire" based on the questions used by Botvinick [4] and Ehrsson [9], which has been repetitively applied in the RHI studies. The applied questions are as follows:

Q1: I felt like I was touching my left hand.

Q2: I felt like my left hand was becoming bigger.

Q3: I felt like my left hand was moving.

The first question $(\mathrm{Q} 1)$ was designed to assess if the participants experienced the RHI. The other two questions $(\mathrm{Q} 2$ and Q3) were unrelated to the RHI and served as a control for the suggestibility. The participants used a seven-point Likert scale, which is a psychometric scale commonly used in questionnaires, to rate the extent to which the statements did or did not apply; in our case, 0 meant "the statement did not apply at all" and 6 meant "I strongly agree with the statement”.

\section{Results and Discussions}

Fig. 6 shows the result of the questionnaire, in which the averaged scores over the 10 participants are illustrated as bar charts; the line segments on the bars indicate its mean errors. Wilcoxon matched pairs (signed-ranked) test, which is a non-parametric test usually used for ordinal scale (ranking scale), was applied to statistically analyze the difference between synchronous and asynchronous conditions for all the statements. In this study, significant effects were reported if $p<0.05$ (5\% significance level). As shown in Fig. 6, only for Q1 score in the synchronous condition were significantly larger than score in the asynchronous condition $(z=2.36$, $p=0.018$ ); in $\mathrm{Q} 2$ and Q3 scores, the null hypothesis (no significant difference) could not be rejected. This is quite similar to the result reported by Ehrsson [9], and implies that in the synchronous condition, the participants had the illusion 
of touching their left hand with their right hand. Q1 scores in the synchronous condition were also significantly larger than synchronous condition scores of Q2 $(z=3.45, p<0.001)$ and Q3 $(z=3.66, p<0.001)$ ruling out the possibility that the scores for Q1 were due to a suggestibility effect. From these results, it is found that the use of the developed masterslave system enables to induce the RHI under the self-touch condition.

\section{CONCLUSIONS}

We have proposed a novel tactile rubber hand illusion (RHI) paradigm for the manipulation of body-parts ownership using haptic technology. The present paper showed that the RHI can be elicited by means of a master-slave system during the synchronous active self-stroking condition; the participants reported the illusion of touching their own left hand with their own right hand although they just brushed a fake rubber hand using a paintbrush with their right hand.

To demonstrate this, first, the self-touch action was characterized by focusing on the tapping force and stroking speed, and the requirements in the master-slave system for the active self-touch were revealed. Then, we illustrated the features of the newly designed master-slave system by showing how it allows studying the self-touch-enabled RHI in an interactive manner. The platform permits not only a precise control of the temporal synchrony and the spatial congruency of the experimentally administered multisensory conflicts, but also the possibility to have force feedback when touching (tapping) one's own body in unusual or impossible anatomical configurations. We strongly believe that the possibility to better manipulate active and passive self-touch as well as to investigate the contribution of haptic feedback to the feeling of body-parts ownership may allow clarifying many current open questions and hypothesis about body representation in human brain. Further, our results would have unlimited potential to open up a new interdisciplinary field between robotics and neuroscience.

\section{ACKNOWLEDGEMENTS}

We thank Roger Gassert in ETH Zurich, Switzerland for giving us an opportunity to start this collaborative study. We also gratefully acknowledge all the participants in our experiment.

\section{REFERENCES}

[1] H. Fabrega, The Feeling of What Happens: Body and Emotion in the Making of Consciousness, A. R. Damasio, Ed. New York, Harcourt Brace \& Company, 1999.

[2] P. Haggard, M. Taylor-Clarke, and S. Kennett, "Tactile perception, cortical representation and the bodily self," Current Biology, vol. 13, no. 5, pp. 170-173, 2003.

[3] M. Jeannerod, "Being oneself," Journal of Physiology-Paris, vol. 101, pp. 161-168, 2007.

[4] M. Botvinick and J. Cohen, "Rubber hands 'feel' touch that eyes see," Nature, vol. 391, p. 756, 1998.

[5] H. H. Ehrsson, C. Spence, and R. E. Passingham, "Thats my hand! activity in premotor cortex reflects feeling of ownershipof a limb," Science, vol. 305, no. 875, pp. 875-877, 2004.

[6] F. Pavani, C. Spence, and J. Driver, "Visual capture of touch: Outof-the-body experiences with rubber gloves," Psychological Science, vol. 11, no. 5, pp. 353-359, 2000.
[7] K. C. Armel and V. S. Ramachandran, "Projecting sensations to external objects: evidence from skin conductance response," Proceedings of the Royal Society of London Series B: Biological Sciences, vol. 270, pp. 1499-1506, 2003.

[8] M. Tsakiris and P. Haggard, "The rubber hand illusion revisited: Visuotactile integration and self-attribution," Journal of Experimental Psychology: Human Perception and Performance, vol. 31, no. 1, pp. 80-91, 2005.

[9] H. H. Ehrsson, N. P. Holmes, and R. E. Passingham, "Touching a rubber hand: Feeling of body ownership is associated with activity in multisensory brain areas," Journal of Neuroscience, vol. 25, no. 45, pp. 10564-10573, 2005.

[10] M. Costantini and P. Haggard, "The rubber hand illusion: Sensitivity and reference frame for body ownership," Consciousness and Cognition, vol. 16, no. 2, pp. 229-240, 2007.

[11] T. Dummer, A. Picot-Annand, T. Neal, and C. Moore, "Movement and the rubber hand illusion," Perception, vol. 38, pp. 271-280, 2009.

[12] R. C. White, A. M. A. Aimola, T. J. Davies, Halleen, and M. Davies, "Tactile expectations and the perception of self-touch: An investigation using the rubber hand paradigm," Consciousness and Cognition, vol. 19, no. 2, pp. 505-519, 2010.

[13] L. Raz, P. L. Weiss, and M. Reiner, The Virtual Hand Illusion and Body Ownership, M. Ferre, Ed. Springer-Verlag Berlin Heidelberg, 2008.

[14] D. Katz, The World of Touch, L. E. LKrueger, Ed. Psychology Press, 1989 (Original work: Der Aufbau der Tastwelt, 1925).

[15] J. J. Gibson, The Senses Considered As Perceptual Systems. Greenwood Press, 1983 (Original work: 1966).

[16] M. P. M. Kammers, F. de Vignemont, L. Verhagen, and H. C. Dijkerman, "The rubber hand illusion in action," Neuropsychologia, vol. 47, pp. 204-211, 2009.

[17] S. J. Blakemore, D. M. Wolpert, and C. D. Frith, "Central cancellation of self-produced tickle sensation," Nature Neuroscience, vol. 1, no. 7, pp. 635-640, 1998. 